\title{
On the Optimum Sampling Time for the Measurement of Pollutants in Ambient Air
}

\author{
Richard J. C. Brown, Daniel Hood, and Andrew S. Brown \\ Analytical Science Team, Quality of life Division, National Physical Laboratory, Teddington, Middlesex TW11 OLW, UK \\ Correspondence should be addressed to Richard J. C. Brown, richard.brown@npl.co.uk
}

Received 14 November 2007; Accepted 23 December 2007

Recommended by Peter B. Stockwell

No measurement result of any kind is complete without an associated statement of uncertainty. In general, the aim is to minimise this measurement uncertainty to the extent that the measurement made is fit for purpose, or the uncertainty meets an acceptable level such as a "data quality objective". This paper demonstrates how the sampling time used to measure a pollutant in ambient air over a given time period, particularly with automatic instruments, can affect the uncertainty of the measurement result. It is shown that the uncertainty of the overall result depends critically on how the precision characteristics of the measuring instrumentation vary with analyte abundance. An example of the determination of these characteristics for mercury vapour measurement and the conclusions that may be drawn about optimum sampling times to minimise uncertainty are presented. For situations where uncertainty is minimised by the use of long sampling periods, the implications of this strategy on the "information richness" of the data and on the detection of short-term episodes, as well as peak concentrations, are discussed.

Copyright (C) 2008 Richard J. C. Brown et al. This is an open access article distributed under the Creative Commons Attribution License, which permits unrestricted use, distribution, and reproduction in any medium, provided the original work is properly cited.

\section{INTRODUCTION}

Air pollution continues to be an issue of great concern to the scientific and medical communities, as well as to members of the general public, the media, and environmental pressure groups [1]. Several requirements drive the need for accurate air quality measurements, including measurement of the exposure of the general population to a variety of toxic compounds, assessment of compliance with legislative limits or similar target values, informing policy development and assessing the effectiveness of abatement strategies, and providing air quality information for the general public and to input into other scientific research. As a result, legislative limits have now been imposed on some elements and compounds in ambient air. An example of this is the EU Air Quality Framework Directive's Fourth Daughter Directive (DD) [2], which requires the measurement of benzo(a)pyrene, $\mathrm{Ni}$, As, and $\mathrm{Cd}$ in the $\mathrm{PM}_{10}$ size fraction (particulates with an aerodynamic diameter of $10 \mu \mathrm{m}$ or less) of ambient air, total gaseous mercury (TGM), and the deposition of $\mathrm{Ni}, \mathrm{As}, \mathrm{Cd}$, and Hg. This DD includes "data quality objectives" that specify requirements for minimum data capture and time coverage of measurements, and for the maximum uncertainties ascribable to the measurement results. Therefore, it is important to work to minimise the uncertainty of these measurements so that they are fit for purpose in this context $[3,4]$. This is particularly important during the development of standard methods $[5,6]$. Many parameters can have an influence on the uncertainty of the measurement of a pollutant concentration over a given time period; among these, sampling time is an important, but rarely considered, variable.

This paper examines the effect of sampling time on the overall measurement uncertainty for automatic instruments that operate by accumulating a pollutant sample for a given period, prior to making a measurement of the collected pollutant in question. Examples of this type of measurement strategy include the trapping of mercury vapour by goldcoated silica traps followed by thermal desorption and measurement with atomic fluorescence, and the trapping of hydrocarbons by polymer-based absorption tubes followed by thermal desorption and measurement with gas chromatography. Whilst the emphasis of this paper is on automatic techniques for air quality monitoring (where there is usually no user intervention during the measurement process), the general principle described here is also extendable to manual measurements (where there is user intervention during the 
measurement process, often between the sampling and analysis phases). It is shown that the uncertainty of the overall result depends critically on how the precision characteristics of the measuring instrumentation vary with analyte abundance.

\section{EXPERIMENTAL}

The principles described in this paper associated with how the precision characteristics of measuring instrumentation vary with analyte abundance have been illustrated using vapour-phase mercury measurements. Sampling for vapourphase mercury took place using low-volume pumps (KNF Neuberger NMP 05) to draw air through "Amasil" (goldcoated silica) adsorption tubes at a rate of $100 \mathrm{~mL} \cdot \mathrm{min}^{-1}$. A very short sampling line consisting of approximately $5 \mathrm{~cm}$ of PTFE tubing connects the adsorption tube to the ambient environment and is preceded by a $0.8 \mu \mathrm{m}$ pore size filter to remove particulates. Analysis of vapour-phase mercury samples takes place using a PS Analytical Sir Galahad II analyser with a fluorescence detector. The instrument is calibrated by the use of a gas-tight syringe, making multiple injections of known masses of mercury vapour onto the permanent absorption tube of the analyser. The known masses of mercury vapour are generated using mercury in glass bell jar apparatus, which allows a saturated vapour of mercury to develop within the air in the bell jar, which is in equilibrium with the atmosphere via a capillary tube [7].

\section{RESULTS AND DISCUSSION}

Consider a given time period $t$, over which an average value for the mass concentration of a pollutant is required. Consider that this measurement is made by an automatic instrument, which accumulates the pollutant over a sampling period (defined by the user), $t_{i}$, prior to measuring the amount, or mass, of collected pollutant. Given that a negligible "changeover" time between samples is assumed, we may write

$$
\frac{t}{t_{i}}=n
$$

where $n$ is the number of sampling periods of equal length during the whole time period. (Where sample "changeover" time is not negligible, or data capture is less than $100 \%$, the requirements of ISO 11222:2002 must also be considered [8].) It is assumed that the dominant uncertainty in such a measurement is the repeatability of the instrumental response; this will be a very good approximation for pollutants at low mass concentrations. It is also assumed that the instrument does not drift significantly over time and that the uncertainty in the volume of air sampled is assumed to be very small in comparison to the analytical step. This is usually a sound approximation, especially for accurately mass flow controlled systems [9]. Moreover, it will be supposed that other systematic uncertainties do not vary with sampling time or pollutant mass concentration. Two different scenarios will be considered: when small variations in mass concentration are observed during the time period $t$, and when

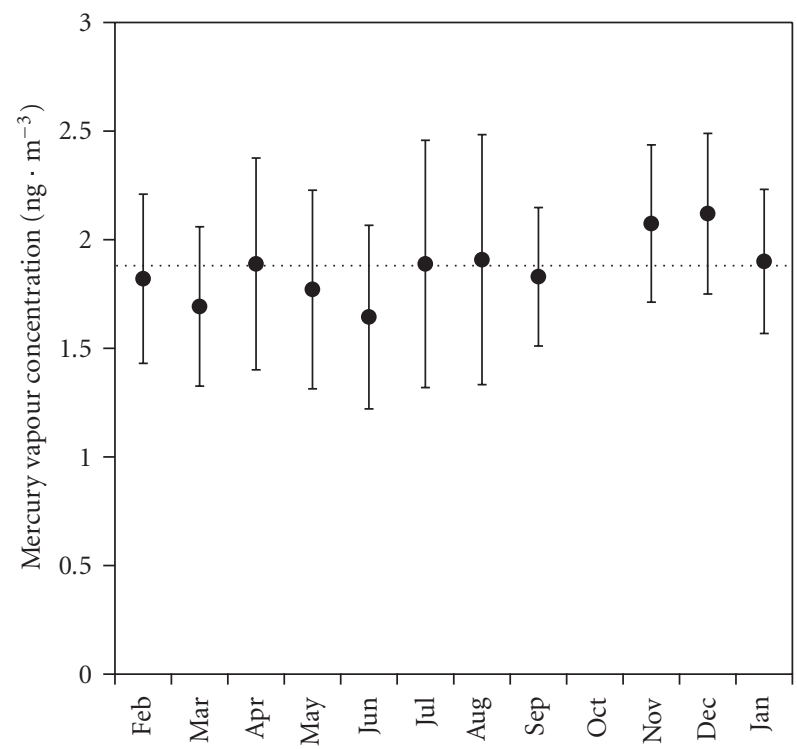

FIGURE 1: The monthly average vapour-phase mercury mass concentration measured at Eskdalemuir, UK, from February 2005 to January 2006. The error bars represent the expanded measurement uncertainty at the $95 \%$ confidence interval. The dotted line represents the unweighted average over this period.

large variations in mass concentration are observed during the time period $t$.

\subsection{Scenarios characterised by small variations in mass concentration during the time period $t$}

In this situation, it is assumed that the mass concentration of the pollutant being measured does not show large variations over the sampling period $t$. Indeed, for the monitoring of vapour-phase mercury in background locations, this is borne out by the results such as in Figure 1, which shows the monthly average vapour-phase mercury mass concentration at Eskdalemuir (a rural background monitoring site) from February 2005 to January 2006 (inclusive). As can be seen, the measured values are all consistent with the yearly average, within the uncertainty of the measurement, over this period.

The average mass concentration, $\bar{\gamma}$, measured over time period, $t$, is given by

$$
\bar{\gamma}=\frac{\sum_{i=1}^{n} \gamma_{i}}{n}
$$

where $\gamma_{i}$ is the average mass concentration measured over sampling time $t_{i}$. The squared standard combined uncertainty in $\bar{\gamma}, u_{c}^{2}(\bar{\gamma})$, is given by

$$
u_{c}^{2}(\bar{\gamma})=\sum_{i=1}^{n}\left[c_{i} \cdot u\left(\gamma_{i}\right)\right]^{2},
$$

where $u\left(\gamma_{i}\right)$ is the uncertainty in $\gamma_{i}$, and $c_{i}$ is the sensitivity coefficient of $\gamma_{i}$. Since

$$
c_{i}=\frac{\delta \bar{\gamma}}{\delta \gamma_{i}}=\frac{1}{n},
$$


it follows that

$$
u_{c}^{2}(\bar{\gamma})=\sum_{i=1}^{n}\left[\frac{u\left(\gamma_{i}\right)}{n}\right]^{2}
$$

If we assume that $\gamma_{i} \approx \gamma_{i+1} \approx \cdots \gamma_{n-1} \approx \gamma_{n}$, then it follows from the assumptions above that $u\left(\gamma_{i}\right) \approx u\left(\gamma_{i+1}\right) \approx$ $\cdots u\left(\gamma_{n-1}\right) \approx u\left(\gamma_{n}\right)$ for a stable analytical instrument. Thus, to a good approximation,

$$
u_{c}^{2}(\bar{\gamma}) \approx n \cdot\left[\frac{u\left(\gamma_{i}\right)}{n}\right]^{2}
$$

so

$$
u_{c}(\bar{\gamma}) \approx \frac{u\left(\gamma_{i}\right)}{\sqrt{n}},
$$

which is a known result for a series of measurements showing little variation $[8,10]$. Assuming that the instrument repeatability is the dominant contribution to uncertainty at low mass concentrations such as those found in ambient air to a first approximation, the uncertainty in $\gamma_{i}$ is given by

$$
u\left(\gamma_{i}\right) \approx \sigma_{i},
$$

where $\sigma_{i}$ is the repeatability of the instrument for the measurement of $\gamma_{i}$. Therefore,

$$
u_{c}(\bar{\gamma}) \approx \frac{\sigma_{i}}{\sqrt{n}} .
$$

Therefore, the relative uncertainty in $\bar{\gamma}, u_{c, \text { rel }}(\bar{\gamma})$, is given by

$$
u_{c, \text { rel }}(\bar{\gamma}) \approx \frac{\sigma_{i, \text { rel }}}{\sqrt{n}},
$$

where $\sigma_{i, \text { rel }}$ is the relative repeatability of the instrument for the measurement of $\gamma_{i}$. At first inspection, it may appear that the relative uncertainty in the mass concentration measured over time period $t$ decreases as $t_{i}$ decreases and, therefore, as $n$ increases. However, this is not necessarily the case, and to understand why, it is necessary to examine the effect of the variation of sampling time on the measurement procedure, and, in particular, how $\sigma_{i, \text { rel }}$ varies with the amount, or mass, of pollutant being measured.

Measuring systems are either heteroscedastic, where the absolute standard deviation of repeat measurements varies as some function of the quantity of analyte being measured or, in the limiting case, homoscedastic, where the absolute standard deviation of repeat measurements does not vary as the quantity of analyte being measured changes. Although most measuring systems are heteroscedastic, over a small range of analyte quantity, they can usually be approximated to homoscedastic behaviour. However, since the sampling times (and therefore the accumulated pollutant mass) under consideration in this study can vary over several orders of magnitude, we must consider that the measuring system will show some heteroscedasticity. Heteroscedastic behaviour can be empirically modelled by a Horwitz-type function (often used to characterise interlaboratory reproducibility) $[11,12]$ of the form

$$
\sigma_{i}=a \cdot m_{i}^{b}
$$

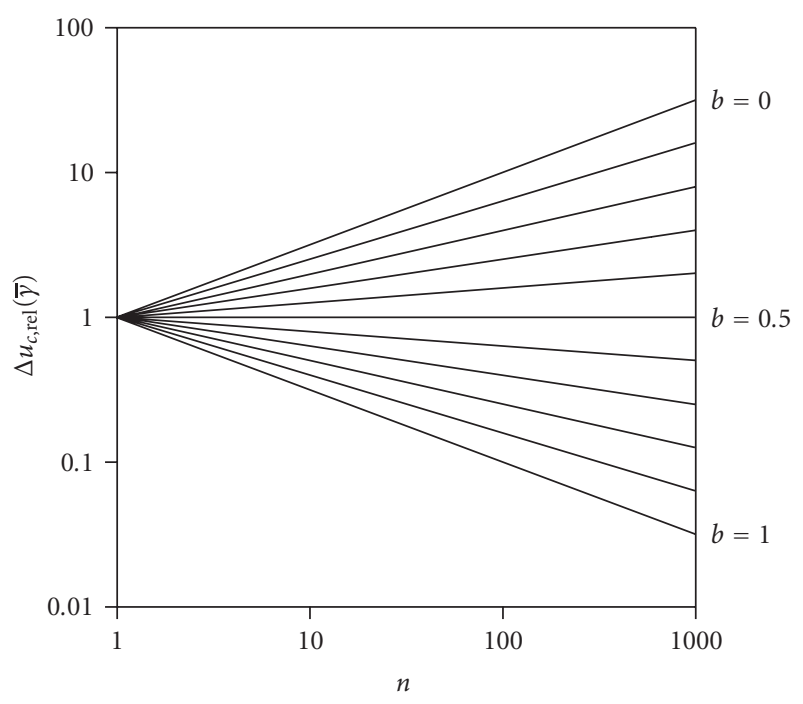

FIGURE 2: The relationship between the expected change in the relative standard uncertainty of the average mass concentration and the number of sampling periods, for various values of $b$ between 0 (top line) and 1 (bottom line), in increments of 0.1 .

Thus,

$$
\sigma_{i, \mathrm{rel}}=a \cdot m_{i}^{(b-1)},
$$

where $a$ and $b$ are constants $(a \geq 0, b \geq 0)$ characterising the individual measuring system in question, and $m_{i}$ is the mass of pollutant (interchangeable for amount, given knowledge of the relative molecular or atomic mass of the pollutant) collected during each sampling period $t_{i}$. If the initial condition of small variations in mass concentration during the time period is met, then the mass of pollutant collected during each sampling period will be approximately proportional to the sampling time, and, therefore, inversely proportional to the number of sampling periods, $m_{i} \propto t_{i} \propto n^{-1}$, such that

$$
\sigma_{i, \mathrm{rel}}=k \cdot \frac{1}{n^{(b-1)}},
$$

where $k$ is a constant similarly characterising the individual measuring system in question. Substituting into (10) gives

$$
u_{c, \text { rel }}(\bar{\gamma}) \approx k \cdot \frac{1}{n^{(b-1)} \sqrt{n}} .
$$

The expected change in $u_{c, \text { rel }}(\bar{\gamma}), \Delta u_{c}$,rel $(\bar{\gamma})$, as the number of sampling periods increases from 1 to $n$, may be expressed as

$$
\Delta u_{c, \text { rel }}(\bar{\gamma}) \approx n^{-b} \cdot \sqrt{n} .
$$

This is simply the ratio of the value of $u_{c, \text { rel }}(\bar{\gamma})$ for $n$ sampling periods to the value of $u_{c, \text { rel }}(\bar{\gamma})$ for 1 sampling period. Equation (15) is plotted for various $b$ in Figure 2.

Figure 2 shows that for a measuring system that is purely homoscedastic, the limiting case where $b=0$, the relative uncertainty of the average mass concentration increases as the number of sampling periods increases. In reality, $b>0$ 
for almost all cases. As $b$ increases, the increase in the uncertainty of the average mass concentration upon increasing the number of sampling periods becomes smaller. When $b=0.5$, the uncertainty of the average mass concentration is independent of the number of sampling periods. For values of $b>0.5$, the relative uncertainty of the average mass concentration begins to fall as the number of sampling periods increases. The exponent $b$ may also take values above 1 , in which case the trend of decreasing uncertainty for increasing numbers of sampling periods would continue.

This analysis has shown that in order to minimise the uncertainty of the average measured mass concentration, it is vital to determine the exponent $b$ for the measurement system in question. As an example, the system used to measure samples on the UK Heavy Metals Monitoring Network [13] — a PS Analytical Sir Galahad II instrument-has been examined to determine how its repeatability characteristics vary with the mass of analyte introduced; this data is presented in Figure 3.

As expected from (12), a plot between normalised relative repeatability and normalised analyte mass on a logarithmic scale, as shown in Figure 3, yields a reasonably linear relationship with a gradient of approximately -0.5 , which is equal to $b-1$, yielding in this case $b \approx 0.5$. Hence, in the case of the Sir Galahad II instrument the data suggests that little difference in the uncertainty of the average mass concentration will be observed by altering the sampling period. Of course, before a decision is made on the optimum number of sampling periods, the characteristics of each measuring device must be determined, as these will vary between instruments and techniques [14].

\subsection{Scenarios characterised by large variations in mass concentration during the time period $t$}

For situations where the quantity being measured exhibits significant changes with time (e.g., at a roadside monitoring site), the uncertainty of the average value of a series on consecutive measurements is, by definition, the square root of the second moment about the mean $[8,10,15]$, which when expressed in a discrete way becomes

$$
u_{c}^{2}(\bar{\gamma})=\frac{1}{n} \sum_{i=1}^{n}\left[u\left(\gamma_{i}\right)\right]^{2} .
$$

In this way, the uncertainty in the average mass concentration value is equal to the square root of the sum of the squared uncertainties associated with the measurements over each sampling period. It is clear, therefore, that the relative uncertainty in the average mass concentration is minimised by minimising the uncertainty of the individual sampling periods. To simplify this situation, we may approximate (16) to

$$
u_{c}(\bar{\gamma}) \approx \bar{u}\left(\gamma_{i}\right)
$$

where $\bar{u}\left(\gamma_{i}\right)$ is the mean uncertainty over all individual sampling periods. (When there is no variation in $u\left(\gamma_{i}\right)$, as in situations characterised by small variations in concentrations,

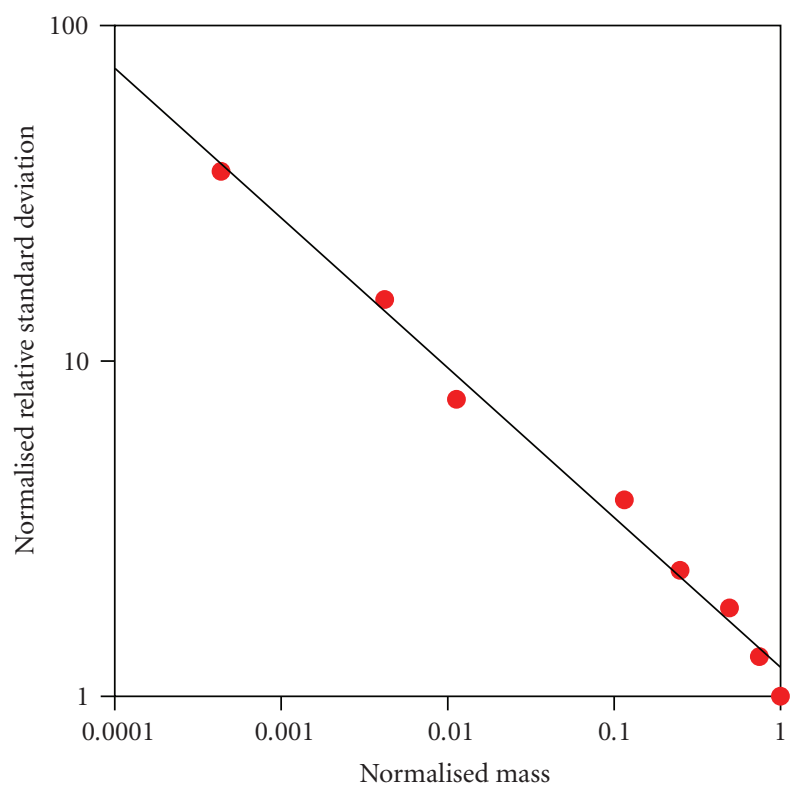

FIgURE 3: The relationship between normalised relative repeatability and normalised analyte mass for the PS Analytical Sir Galahad II instrument.

this approximation becomes an equality.) Unlike (7), there is no dependence on $n$ at this stage. Similarly to (10),

$$
\begin{aligned}
u_{c}(\bar{\gamma}) & \approx \bar{\sigma}_{i}, \\
u_{c, \text { rel }}(\bar{\gamma}) & \approx \bar{\sigma}_{i, \text { rel }},
\end{aligned}
$$

where $\bar{\sigma}_{i}$ and $\bar{\sigma}_{i \text {,rel }}$ are the average repeatability, and average relative repeatability, respectively, of the instrument over all sampling periods. Thus, similarly to (10) to (15), it follows that

$$
\Delta u_{c, \text { rel }}(\bar{\gamma}) \approx n^{1-b}
$$

Equation (19) is plotted in Figure 4.

For the situation indicated in Figure 4, for $b<1$, the relative uncertainty of the average mass concentration increases as the number of sampling periods within the time period increases. Therefore, the uncertainty in this situation would be minimised by using as few sampling periods as possible. When $b=1$, the relative uncertainty of the average mass concentration is independent of the number of sampling periods. Only for situations when $b>1$ is the uncertainty minimised by increasing the number of sampling periods.

\subsection{The effect of sampling time on the information contained within the time series}

In the circumstances described above, decreasing the number of sampling periods can, under some circumstances, decrease the uncertainty of the average measured concentration. However, this is at the expense of the information contained within the time series data. Clearly, the number of data points obtained during the time period, $t$, is directly 


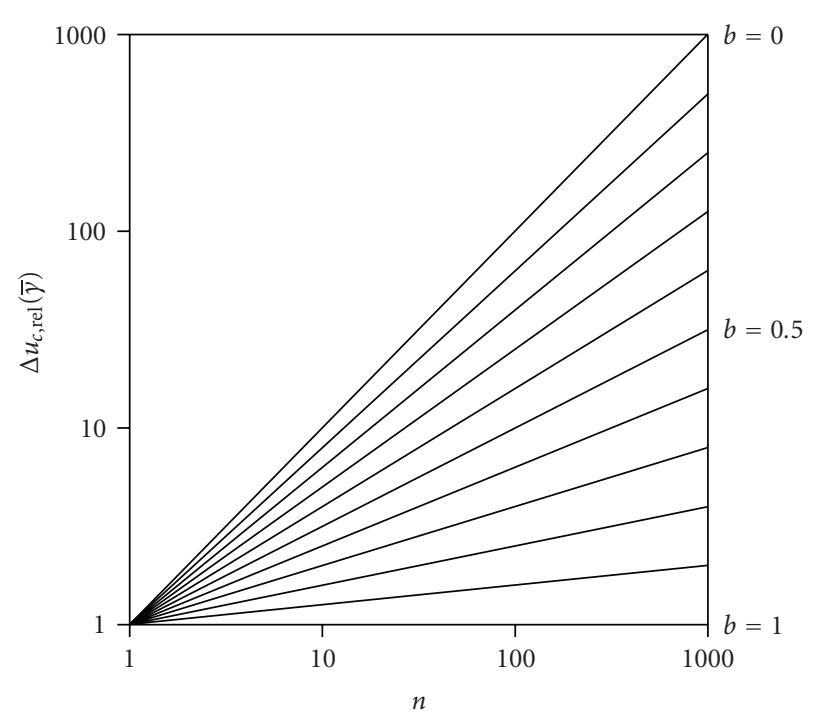

FIGURE 4: The relationship between the change in the relative standard uncertainty of the average mass concentration and the number of sampling periods, for various values of $b$ between 0 (top line) and 1 (bottom line, coincident with the abscissa), in increments of 0.1 .

proportional to the number of sampling periods, $n$. Whilst the average of the data over the time period, $t$, will remain unchanged, the detail of the concentration fluctuations will be lost, especially short-term, high-concentration peaks. This effect is displayed in Figure 5 for $\mathrm{PM}_{10}$ mass concentration at Marylebone Road in London during 2006 [16], which shows how the detail in the data is lost as the number of sampling periods decreases. This is further evidenced by the decrease in the relative standard deviation of the data, and the peak mass concentration, as the sampling period increases, shown in Figure 6.

Obviously for some applications such as odour monitoring [17], determination of short-term peak concentrations is important. The same is true for $\mathrm{PM}_{10}$ measurements, where limits are set not only for annual average concentrations, but also for the number of 24-hour averages above a threshold level [18]. Knowledge of short-term peak concentrations can also be of great value in air pollution modelling [19]. However, the case is different for those compounds for which average exposure over long periods of time, rather than acute exposure over very short periods, is thought to be most important, or for those compounds whose effect is cumulative such as mercury. In these situations, it is better to have data with the lowest uncertainty so that health studies, abatement strategies, and air quality policy can be best assessed and informed.

\section{CONCLUSIONS}

This paper has described how the uncertainty of a time series average can vary as a function of the length of the individual sampling periods. The average of the time series will remain constant regardless of sampling periods. The uncertainty of the average has been investigated for scenarios, where the

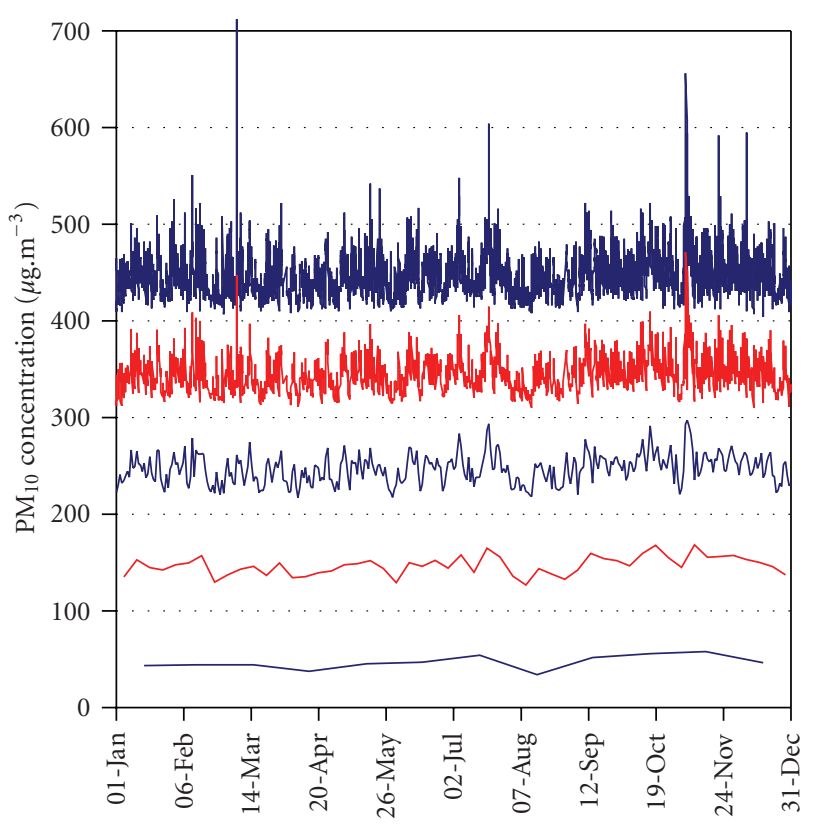

FIgURE 5: The measured $\mathrm{PM}_{10}$ mass concentration at Marylebone Road in London during 2006 displayed, from top to bottom: hourly (offset by $+400 \mu \mathrm{g} \cdot \mathrm{m}^{-3}$ ), six-hourly (offset by $+300 \mu \mathrm{g} \cdot \mathrm{m}^{-3}$ ), daily (offset by $+200 \mu \mathrm{g} \cdot \mathrm{m}^{-3}$ ), weekly (offset by $+100 \mu \mathrm{g} \cdot \mathrm{m}^{-3}$ ), and monthly no soffset.

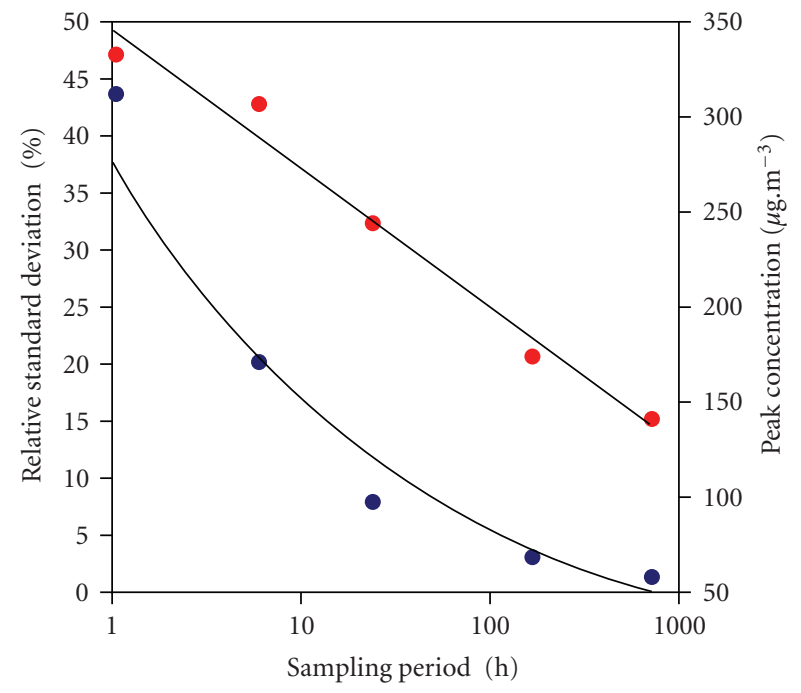

Figure 6: The variation in relative standard deviation (red bullets) and peak concentration (blue bullets) of measured $\mathrm{PM}_{10}$ mass concentrations at Marylebone Road, London, 2006, with changing sampling periods.

pollutant concentration is not expected to vary significantly, and for scenarios where a larger variation in concentration is expected. It has been shown that the outcome is crucially dependent on how the precision characteristics of the measuring instrumentation vary with analyte abundance. For the example of vapour-phase mercury measurement on the UK 
Heavy Metals Monitoring Network, it has been shown that the uncertainty of the average is not strongly affected by sampling time.

The choice of sampling period is a balance between achieving fit-for-purpose data with an acceptable uncertainty, and the requirements for the use of the measurement data.Generally, one should act so as to minimise the uncertainty of the measurement data; and in some cases, this will require longer sampling periods to be used. This is acceptable for the measurement of pollutants, which cause adverse health effects by cumulative or long-term, low-level exposure. Many such pollutants are only required by legislation to be reported on an annual average basis, and thus long sampling periods can be easily accommodated. However, as has been observed, the use of long sampling periods has a detrimental effect on the information richness of the measurement data. For pollutants where adverse health effectsare caused by the peak concentration over short periods, or where legislation demands short-term average data to be reported, or sampling campaigns designed to measure shortterm peak concentrations of any pollutant, long sampling periods are not acceptable. In these cases, the choice of sampling periods must be balanced between meeting these demands and delivering fit-for-purpose uncertainties.

\section{ACKNOWLEDGEMENT}

The funding of this work by the UK DIUS National Measurement System's Chemical and Biological Metrology Knowledge Base Programme is gratefully acknowledged.

\section{REFERENCES}

[1] D. Vallero, Fundamentals of Air Pollution, Academic Press, London, UK, 4th edition, 2007.

[2] European Commission DG Environment, "Council Directive 2004/107/EC of the European Parliament and of the Council of 15 December 2004 relating to arsenic, cadmium, mercury, nickel and polycyclic aromatic hydrocarbons in ambient air," Official Journal of the European Union, vol. L023, pp. 3-16, 2005.

[3] R. J. C. Brown and M. J. T. Milton, "Developments in accurate and traceable chemical measurements," Chemical Society Reviews, vol. 36, no. 6, pp. 904-913, 2007.

[4] R. J. C. Brown and M. J. T. Milton, "Analytical techniques for trace element analysis: an overview," Trends in Analytical Chemistry, vol. 24, no. 3, pp. 266-274, 2005.

[5] E. J. Sneek, "Type approval of instruments and uncertainty calculations in the new EN-standards for the measurement of $\mathrm{SO}_{2}, \mathrm{NO}_{2}, \mathrm{CO}$ and $\mathrm{O}_{3}$ in ambient air," Gefahrstoffe Reinhaltung Der Luft, vol. 63, no. 4, pp. 163-166, 2003.

[6] K. Berger, F. Bunzel, and R. Neuroth, "Standardisation of a European reference measurement method for the determination of $\mathrm{Pb}, \mathrm{As}, \mathrm{Cd}$ and $\mathrm{Ni}$ in ambient air-validation and determination of the measurement uncertainty," Gefahrstoffe Reinhaltung Der Luft, vol. 63, no. 4, pp. 157-162, 2003.

[7] R. Dumarey, E. Temmerman, R. Dams, and J. Hoste, "The accuracy of the vapour-injection calibration method for the determination of mercury by amalgamation/cold-vapour atomic absorption spectrometry," Analytica Chimica Acta, vol. 170, pp. 337-340, 1985.
[8] ISO 11222:2002, "Air quality-determination of the uncertainty of the time average of air quality measurement," ISO, Geneva, 2002.

[9] S. S. Wulff and M. A. Weitz, "Measurement uncertainty in the calibration of low-flow ambient air samplers," Quality and Reliability Engineering International, vol. 21, no. 6, pp. 605-619, 2005.

[10] P. P. Ballesta, "The uncertainty of averaging a time series of measurements and its use in environmental legislation," Atmospheric Environment, vol. 39, no. 11, pp. 2003-2009, 2005.

[11] M. Thompson and P. J. Lowthian, "A Horwitz-like function describes precision in a proficiency test," The Analyst, vol. 120, pp. 271-272, 1995.

[12] M. Thompson and P. J. Lowthian, "The Horwitz function revisited," Journal of AOAC International, vol. 80, no. 3, pp. 676679, 1997.

[13] R. J. C. Brown, R. E. Yardley, D. Muhunthan, et al., "Twentyfive years of nationwide ambient metals measurement in the United Kingdom: concentration levels and trends," to appear in Environmental Monitoring and Assessment 2008.

[14] M. Thompson and R. Wood, "Using uncertainty functions to predict and specify the performance of analytical methods," Accreditation and Quality Assurance, vol. 10, no. 9, pp. 471478, 2006.

[15] C. F. Dietrich, "The statistics of scientific and industrial measurement," in Uncertainty, Calibration and Probability, Adam Hilger, Bristol, UK, 2nd edition, 1991.

[16] http://www.airquality.co.uk/, September 2007.

[17] G. H. Drew, R. Smith, V. Gerard, et al., "Appropriateness of selecting different averaging times for modelling chronic and acute exposure to environmental odours," Atmospheric Environment, vol. 41, no. 13, pp. 2870-2880, 2007.

[18] European Commission DG Environment, "Council Directive 1999/30/EC of the European Parliament and of the Council of 22 April 1999 relating to limit values for sulphur dioxide, nitrogen dioxide and oxides of nitrogen, particulate matter and lead in ambient air," Official Journal of the European Communities, vol. L163, pp. 41-60, 1999.

[19] A. Venkatram, "Accounting for averaging time in air pollution modelling," Atmospheric Environment, vol. 36, no. 13, pp. 2165-2170, 2002. 


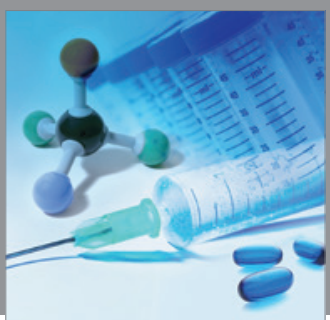

International Journal of

Medicinal Chemistry

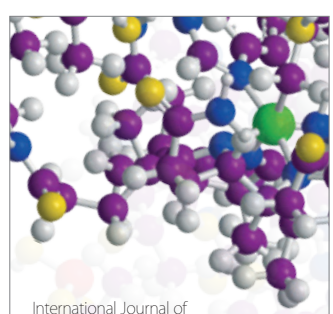

Carbohydrate Chemistry

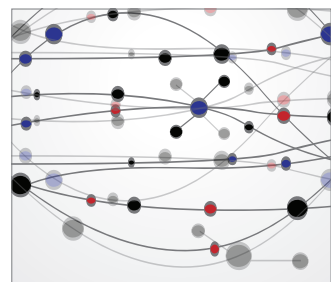

The Scientific World Journal
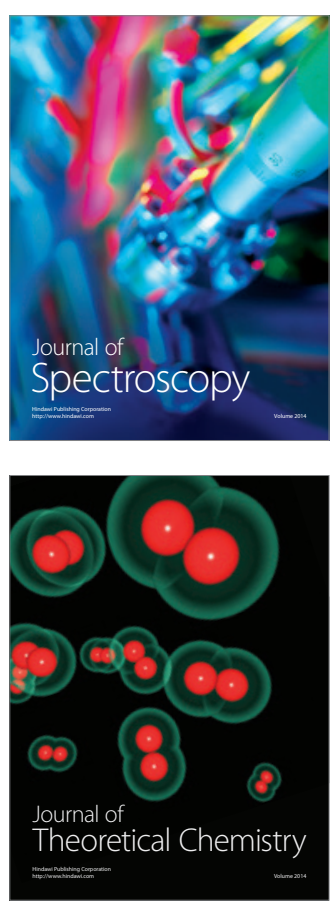
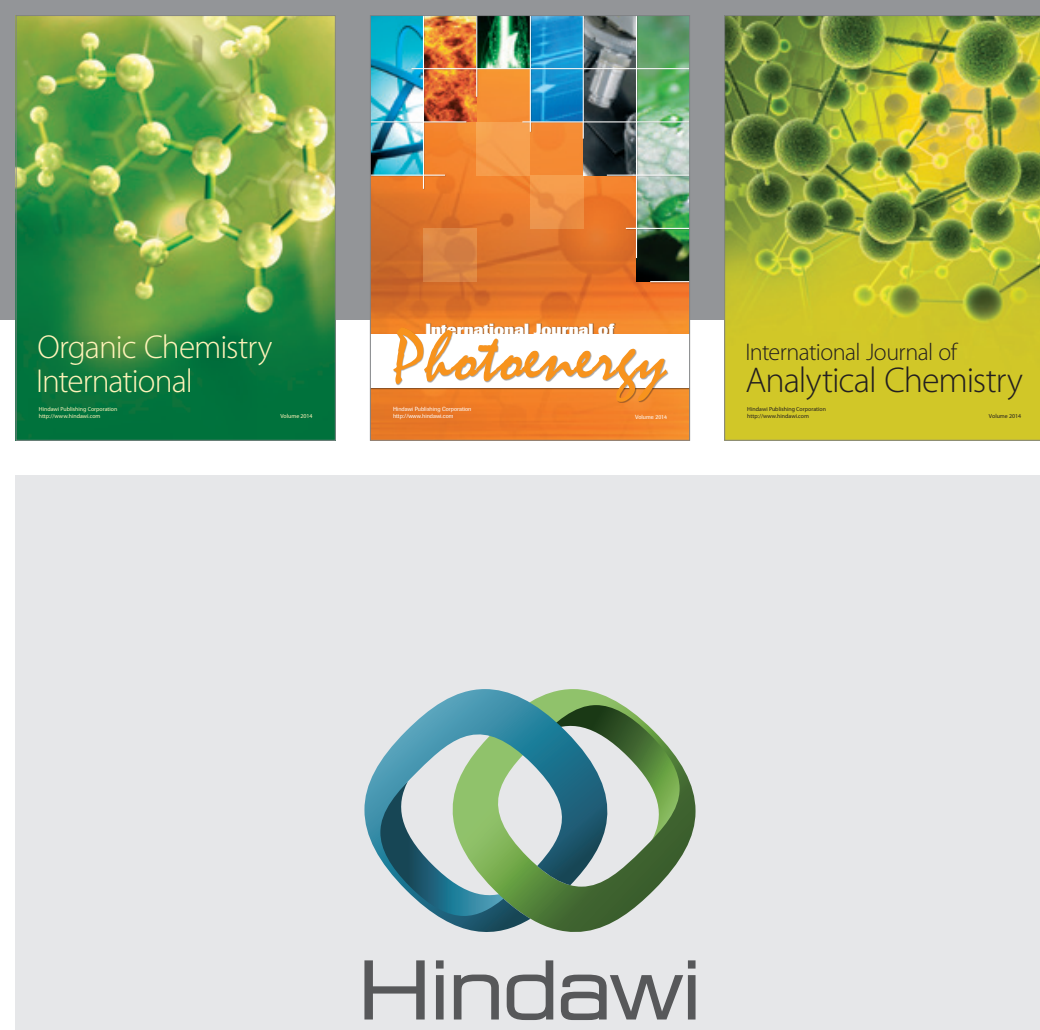

Submit your manuscripts at

http://www.hindawi.com
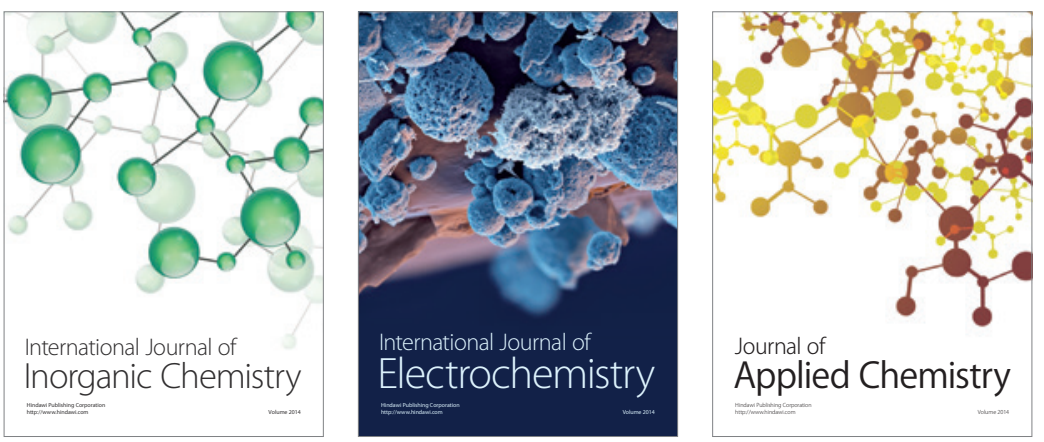

Journal of

Applied Chemistry
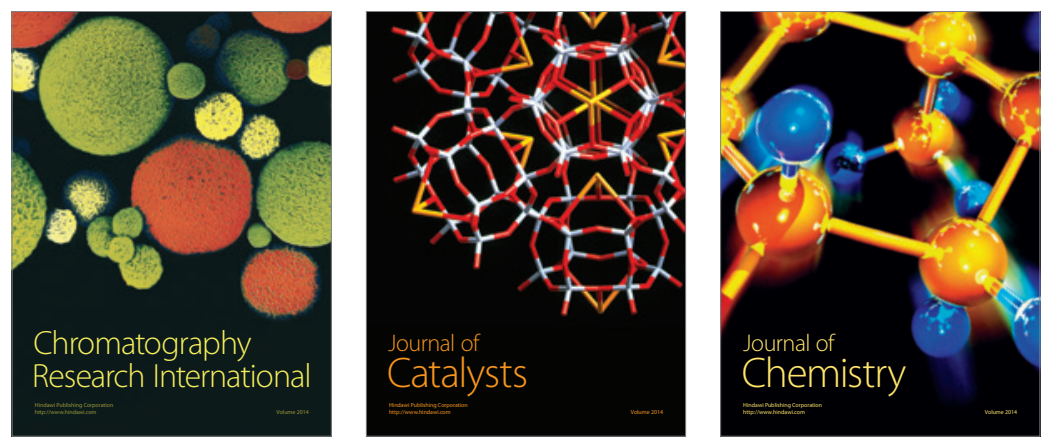
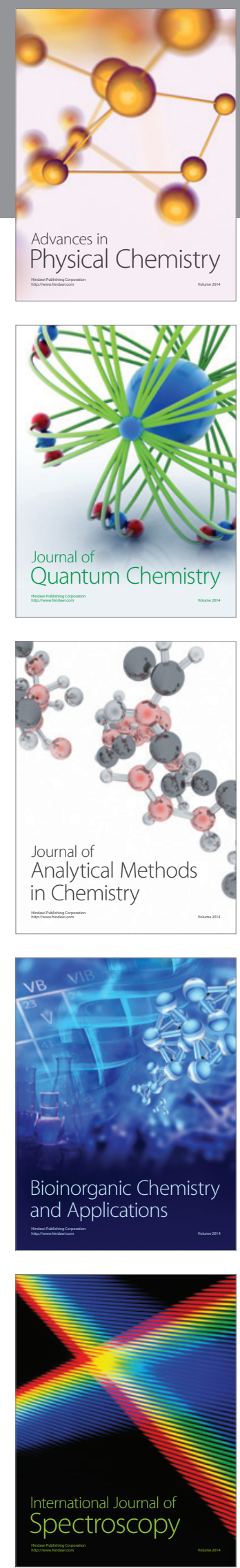